\title{
Rectal cancer: latest developments and innovative new treatment concepts
}

\author{
Arno Amann
}

(C) Springer-Verlag GmbH Austria, part of Springer Nature 2020

In this special issue of the Magazine of European Medical Oncology (MEMO), a multidisciplinary team of experts summarizes and discusses recent developments and interesting results in rectum carcinoma, from a rising incidence in a young patient cohort to neoadjuvant, palliative and adjuvant treatment protocols. Especially up-front therapy before surgery has been the field within the multimodal treatment of this cancer entity with the most promising and interesting developments over the last year, raising the question of whether surgery is always needed or whether an organ-preserving concept will be the way to go in future.

First, the retrospective study by Zaid et al. [1] evaluates the clinicopathological features and outcomes of an increasing rectal cancer population in young Egyptian patients (<40 years). In this special subpopulation, a more aggressive histophenotype (signet ring cell cancer) was found and a clear correlation between the completion of neoadjuvant treatments and disease free survival (DFS)/overall survival (OS) was found.

Lunger at al. [2] discuss the role of chemoradiotherapy alone or chemoradiotherapy followed by surgery in rectal cancer. They conclude that in selected patients with a complete response after neoadjuvant treatment a watch and wait and thereby organ-preserving approach could be a feasible way for the future, preserving foremost quality of life in young and fit patients.

In the next article, by Arnold et al. [3], novel approaches with special regard to novel treatment

\section{A. Amann, MD ( $ه)$}

Department of Hematology and Oncology, Internal

Medicine V, Medical University of Innsbruck,

Anichstraße 35, 6020 Innsbruck, Austria

arno.amann@tirol-kliniken.at combinations in neoadjuvant chemoradiotherapy concerning rectal cancer are discussed. Three innovative and promising new strategies to improve rectal cancer treatment, the total neoadjuvant therapy, short course radiotherapy and the implementation of immune checkpoint inhibitors seem to be the way to go in the future.

In the short review by Reichinger et al. [4], a novel form of guided adjuvant treatment in colorectal cancer is discussed by analysing circulating tumour DNA (ctDNA) as an indicator of residual disease after surgery or as a prognostic factor for early relapse. As a result, ctDNA could be a tool to select high-risk subgroups in advanced colorectal cancer (CRC) and help to select the right adjuvant treatment protocol.

In the final article, Witte et al. [5] summarize an interesting case report of a young female patient with sigmoid colon cancer and synchronous pulmonary metastasis during four lines of immunochemotherapy combinations. Thereby, the authors highlight the crucial role of retaining a high-intensity treatment approach throughout the disease for selected fit patients to maintain disease control.

In conclusion, a multimodality treatment approach is of course still the way to go in treating patients with colorectal cancer. In my opinion, it is probably not yet the time to alter long unchanged protocols especially in neoadjuvant treatment of rectal cancer, but if more data becomes available a major change will be seen here. As a guest editor I hope the summary of these articles helps to maintain and raise interest in the topic of rectal cancer.

With best regards,

Arno Amann MD 


\section{editorial}

Conflict of interest A. Amann declares that he has no competing interests.

\section{References}

1. Zaid AM, Aboelnaga EM, Halim A, Abdelkhalek M, Elbalka SS, Zuhdy M, Fareed AM, Ibrahim EM, Halim H, Metwally IH. Rectal cancer among younger Egyptian patients-clinico-pathological features and oncologic outcomes: A single institution experience. memo 2020. https://doi.org/10.1007/s12254-020-00622-z.

2. Lunger F, Peros G. Chemoradiotherapy alone or chemoradiotherapy followed by surgery in rectal cancer. Which way to go? memo. 2020. https://doi.org/10.1007/s12254-02000586-0.

3. ArnoldCR, Mangesius J, JägerR, GanswindtU. Neoadjuvant chemoradiotherapy in rectal cancer. Are there new drug combinations on the horizon? memo. 2020. https://doi. org/10.1007/s12254-020-00594-0.
4. Reichinger A, Rumpold H. Circulating tumour DNA-guided adjuvant chemotherapy in colorectal carcinoma. memo. 2020. https://doi.org/10.1007/s12254-020-00607-y.

5. Witte FH, Hilbe W, Müldür E. Four lines of immunochemotherapy combinations in a young patient with an aggressive metastatic colorectal cancer. memo. 2020. https://doi.org/ $10.1007 / \mathrm{s} 12254-020-00627-8$.

Publisher's Note Springer Nature remains neutral with regard to jurisdictional claims in published maps and institutional affiliations.

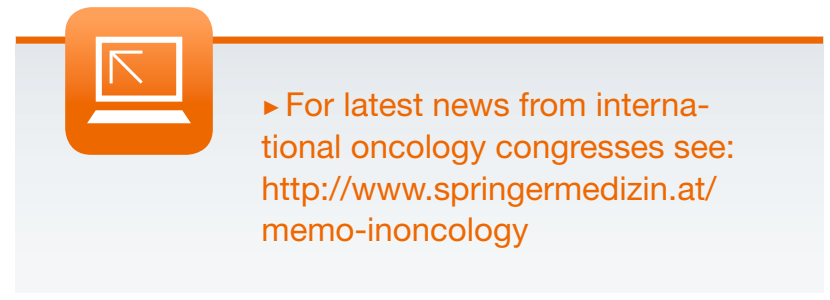

